\title{
Bioactive Polysaccharides Based Graphene Oxide Nanoparticle as a Promising Carrier for Anticancer Drug Delivery
}

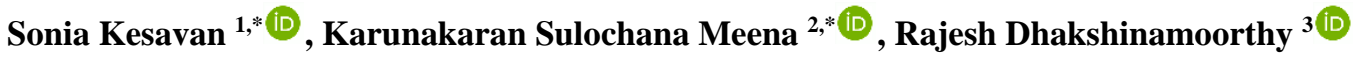 \\ 1 Department of Chemistry, Queen Mary's College (Affiliated to University of Madras), Chennai-600 004, Tamilnadu, \\ India; soniakesavanmc2@gmail.com (S.K); \\ 2 Alagappa Government Arts College, Karaikudi- 630 003, Tamilnadu, India; meenamohan90@ gmail.com (K.S.M); \\ 3 Department of Microbiology, Chennai National Arts and Science College(Affiliated to University of Madras), Chennai - \\ 600 054, Tamilnadu, India; rajesh.d975@gmail.com (R.D); \\ * Correspondence: soniakesavanmc2@gmail.com (S.K); meenamohan90@gmail.com (K.S.M); \\ Scopus Author ID 57194725278
}

Received: 17.06.2021; Revised: 25.07.2021; Accepted: 29.07.2021; Published: 8.08.2021

\begin{abstract}
Nowadays, the concept of drug transmission is a prominent issue in the world of drug delivery research. We investigated the development of a hybrid platform based on graphene oxide/chitosan and xyloglucan (GO-CH-Xn) for the loading and release of doxorubicin (DOX)., where chitosan (CS) natural polymer functionalizes graphene oxide and is then grafted by xyloglucan (Xn) natural hydrophilic polysaccharide to form a reliable nanocarrier system for the delivery of DOX. UVVis spectroscopy, Fourier transform infrared spectroscopy, X-ray diffraction, Raman spectroscopy, transmission electron microscopy, and scanning electron microscopy analysis were used to confirm the fundamental physicochemical properties. The DOX loading capacity and efficiency were $81.8 \%$ and $73.5 \%$. The graphene oxide-chitosan-xyloglucan- doxorubicin (GO-CS-Xn-DOX) drug delivery system showed a pH-regulated release as observed by UV analysis. Biocompatibility was evaluated via in vitro hemolysis assay, indicates negligible toxicity, and the anticancer activity of the developed nanocarrier system was studied by 3-(4, 5-dimethylthiazol-2-Y)-2,5-diphenyltetrazolium bromide (MTT) against human (U 87) glioblastoma cancer cell lines. The in vitro studies demonstrate the major advantages of the developed approach by demonstrating its capability as a promising nanocarrier for biomedical applications.
\end{abstract}

Keywords: graphene oxide; xyloglucan; chitosan; drug delivery; anticancer activity.

(C) 2021 by the authors. This article is an open-access article distributed under the terms and conditions of the Creative Commons Attribution (CC BY) license (https://creativecommons.org/licenses/by/4.0/).

\section{Introduction}

Nowadays, the scientific community's current focuses are developing a selective drug delivery system from natural polymeric material for cancer therapeutics. Currently, cancer is the major health threat worldwide and is treated using chemotherapy, nanotherapeutics, and gene therapy $[1,2]$. Current tumor drug delivery strategies are centered on increasing drug concentration at the target site while decreasing systematic distribution. [3]. In this view, functionalized nanoparticles are of great interest since they can prevent the systemic metabolism and successive elimination of the drug, thus assuring pharmacological defects with less toxicity [3, 4]. Numerous studies are focused on the surface functionalization of nanoparticles to increase the retention time of nanoparticles. Drug nanocarriers can also be 
viewed as a bridge between nanotechnology and delivery systems, and they play an important role in clinical cancer therapy. Among different types of nanocarriers, graphene-based derivatives have gained much attention due to their two-dimensional nanostructure, high thermal, electrical conductivity, surface area, number of layers, and lower toxicity. All these properties make graphene unique from other nanocarriers [5-7]. The physicochemical properties of graphene, such as electrical and thermal conductivity, optical transparency, and mechanical strength can be easily changed by chemical functionalization [8,9].

Graphene oxide (GO), the oxidized form of graphene, a two-dimensional carbon material, contains several hydrophilic functional groups such as hydroxyl, epoxy, and carboxylic groups on the basal plane and its edges [10]. In biomedical fields such as drug delivery [11], biosensing [12], bioimaging [13], cancer therapy [14], tissue engineering [15], and wound healing [16], graphene and its analogs, such as GO, reduced graphene oxide (rGO), and graphene sheets, have sparked a lot of interest. GO and GO-based nanocomposites has the potential to be an effective nanocarrier for the delivery of anticancer drugs such as Doxorubicin [17], Sumatriptan succinate [18], Methotrexate [19], Quercetin and gefitinib [20], Proanthocyanidins [21], Cisplatin/ DOX [22], 5-fluorouracil/Curcumin [23,24], Camptothecin [25] and cytarabine [26]. The large surface area and presence of $\pi$ electrons on the GO surface enable $\pi-\pi$ interactions leading to the high loading capacity of the drug.

Recent developments in the use of polysaccharide bio nanocomposite along with GO in pharmacological applications have been considered [27]. Materials that respond to stimuli, such as GO, chitosan, cellulose, albumin, and gelatin, have widely applied in sensors [28], actuators [29], self-healing coatings [30], textiles [31], diagnostics [32], soft robots [33], and optical systems [34].

Diverse natural polymers have been utilized as raw materials [35] for the functionalization of GO since they provide great advantages to drug targeting, delivery, and release, thus exhibits a prominent role in nanomedicine. The functionalization of GO with biocompatible polymers increases the stability, biocompatibility, and drug loading capacity. GO was functionalized with Chitosan (CS), a natural polycationic amino polysaccharide consists of randomly distributed glucosamine and $\mathrm{N}$-acetyl glucosamine obtained from chitin upon partial deacetylation [35-37]. It has been explored widely for biomedical applications such as bone/skin regeneration, wound dressing, and cancer therapy [38, 39]

Natural polymers/polysaccharides are frequently investigated for use in drug delivery systems due to their $\mathrm{pH}$ response capacity, biodegradability, biocompatibility, nontoxicity, abundance in nature, and adaptability to chemical modifications. [40-42]. In this view, Xyloglucan (Xn), also a natural polymer obtained from tamarind seeds, is a neutral hemicelluloses polysaccharide comprised of glucopyranose residues with xylo and galacto pyranose residues $[43,44]$. In drug delivery applications, Xn has been reported as an appealing and functional natural polysaccharide [44, 45]. More research on xyloglucan-based drug delivery systems has recently been conducted, including nanoagrregrates, nanospheres, hydrogels, and films for biomedical applications [45]. Hence this polysaccharide possess great versatility and should be studied by research experts. The goal of this research is to create a novel nanohybrid formulation for the loading of DOX. However, the solubility and biocompatibility of GO were relatively low and weak. To improve GO properties, it was functionalized with CS and then grated with $\mathrm{Xn}$, resulting in the formation of the nanocarrier 
GO-CS-Xn, which not only improves the solubility and biocompatibility of GO, but also improves the drug loading capacity.

When assessing the efficacy of a designed drug delivery system, the nanocarrier and loaded drugs should be considered. As a model drug, doxorubicin (DOX), an effective therapeutic agent, was used. Furthermore, xyloglucan (Xn) has not been incorporated into the GO-CS composite, nor has its anticancer activity been investigated. As a result, the current study was implemented. By functionalizing the GO surface with CS via an amide bond, we developed a novel nanohybrid platform. Then Xn was grafted to CS in the GO-CS composites via amidation. The modification was analyzed using UV-Visible, FT-IR, XRD, Raman spectroscopy, TEM, SEM, and zeta potential. The comportment of GO-CS-Xn in the loading and release of chemotherapeutic drug DOX was investigated by UV-Vis spectroscopy. Human red blood cell rupture was evaluated by hemolysis assay. The cell-killing capability of GO-CS$\mathrm{Xn}$ and GO-CS-Xn-DOX in inhibiting the growth of cancer cells was evaluated using U87 cells to show the effectiveness of the nanocarriers. It is believed that this hybrid nano platform can have great prospective as a targeted drug delivery system.

\section{Materials and Methods}

\subsection{Materials.}

Graphite powder, sulphuric acid $\left(\mathrm{H}_{2} \mathrm{SO}_{4}\right)$, hydrochloric acid $(\mathrm{HCl})$, sodium nitrate $\left(\mathrm{NaNO}_{3}\right), \mathrm{N}$-hydroxysuccinimide (NHS), potassium permanganate $\left(\mathrm{KMnO}_{4}\right), 1$-ethyl-3-[3(dimethylamino)propyl]carbodiimide hydrochloride (EDC), 30\% hydrogen peroxide $\left(\mathrm{H}_{2} \mathrm{O}_{2}\right)$ and ethanol were obtained from SRL; Chitosan (90\% deacetylation) was purchased from Marine Hydrocolloid. Xyloglucan from Tamarind seed powder was obtained as a gift sample from Indra Agrotech Private Ltd (Maharashtra, India).Human glioblastoma cell line (U87) from National Centre for Cell Science (NCCS), Pune, India. The 10\% fetal bovine serum (FBS), DMEM medium, and antibiotics were purchased from HiMedia, India.

\subsection{Preparation of graphene oxide $(G O)$.}

GO was prepared through modified Hummer's method [46,47] describes as follows: $1 \mathrm{~g}$ of graphite powder was dispersed into a mixture of $30 \mathrm{ml}$ of concentrated sulphuric acid and $0.75 \mathrm{~g}$ of sodium nitrate in an ice bath. Then, while stirring, $3 \mathrm{~g}$ of potassium permanganate was gradually added to the resulting mixture. The reaction mixture was then stirred at $40^{\circ} \mathrm{C}$ for $24 \mathrm{~h}$. After $24 \mathrm{~h}$ the reaction was terminated by adding distilled water followed by $20 \mathrm{ml}$ of $30 \%$ hydrogen peroxide. The final product GO was collected and washed with dilute hydrochloric acid and water until the solution becomes neutral. Finally, an aqueous suspension of GO was sonicated for $1 \mathrm{~h}$. The resultant GO was then lyophilized and stored in vials for further use.

\subsection{Preparation of functionalized $G O$ with $C S$.}

CS was conjugated onto the surface of GO via amidation in the presence of EDC and NHS, where the carboxyl group of GO reacts with the amino groups of CS, resulting in the formation of an amide bond. The synthesis process was employed as follows: GO $(30 \mathrm{ml}$; $1 \mathrm{mg} / \mathrm{ml}$ ) was sonicated for $1 \mathrm{~h}, 150 \mathrm{mg}$ of EDC, and $300 \mathrm{mg}$ of NHS were added gradually to $\mathrm{GO}$ suspension, activating the $-\mathrm{COOH}$ group of $\mathrm{GO}$ and kept under constant stirring. To this 
CS in $1 \%$,the acetic acid solution was added slowly and stirred for $24 \mathrm{~h}$. The GO-CS product was thoroughly washed with water to removed unreacted CS. The final solution was lyophilized to obtain GO-CS powder.

\subsection{Preparation of GO-CS-Xn nanocomposite.}

GO-CS-Xn composite was typically prepared as follows: Firstly, 200mg of GO-CS were dispersed in $100 \mathrm{ml}$ of distilled water at room temperature. Then, $75 \mathrm{mg}$ of CDI and $100 \mathrm{mg}$ $(50 \mathrm{ml})$ of $\mathrm{Xn}$ solution were added to the suspension and stirred for $5 \mathrm{~h}$ at room temperature. Finally, the resultant mixture was centrifuged at $10,000 \mathrm{rpm}$ for $10 \mathrm{~min}$, and the precipitant was washed several times with distilled water and freeze-dried for $24 \mathrm{~h}$.

\subsection{Instrumentation.}

UV- Vis Spectra was recorded using Perkin Elmer Lambda 35 Spectrometer.The FTIR spectra of synthesized graphene-based nanocarriers were done with a Perkin Elmer (spectrum two models) over the range of 400-4000 $\mathrm{cm}^{-1}$.X-ray diffraction (XRD) was recorded using a Bruker, Germany $\mathrm{D} 8$ advance $(\mathrm{Cu} \mathrm{K \alpha}$ and Ni-filtered radiation). Raman spectroscopy was recorded from 500 to $3500 \mathrm{~cm}^{-1}$ Bruker RFS27: Standalone FT-Raman Spectrometer at a wavelength of $532 \mathrm{~nm}$. Surface charge and size distribution were measured using Horiba SZ 100 Nanopartica. Scanning electron microscopy (Carl Zeiss Evo 18 model) and Transmission electron microscopy (FEI Tecnai, G2 20Twin) were used to analyze the samples' morphology.

\subsection{Loading of DOX onto GO-CS-Xn nanocomposite.}

$0.50 \mathrm{mg}$ of GO-CS-Xn was dispersed in $1 \mathrm{~mL}$ of distilled water. To this, $5 \mathrm{ml}$ of DOX solution at a concentration of $1 \mathrm{mg} / \mathrm{mL}$ was added, and the mixture was kept under stirring for $48 \mathrm{~h}$ in the dark at room temperature. The resultant solution was centrifuged at 10,000rpm for $20 \mathrm{~min}$. The concentration of DOX in the supernatant solution was measured using a UV-Vis spectrophotometer at the wavelength of $480 \mathrm{~nm}$. The loading capacity and efficiency of DOX on the GO-CS-Xn nanocarrier were determined as follows:

$$
\begin{gathered}
\text { DOX loading capacity }(\%)=\frac{\text { Weight of loaded DOX }}{\text { Weight of nanocarrier }} \times 100 \\
\text { DOX loading capacity }(\%)=\frac{\text { Initial DOX conc. - Supernatant DOX conc. }}{\text { Initial DOX conc. }} \times 100
\end{gathered}
$$

\subsection{In vitro hemolysis assay.}

The in vitro hemolysis assay was performed according to the Muthukumarasamyvel et al. method [48]. In brief, $3 \mathrm{~mL}$ of fresh human blood was collected, washed thrice with PBS, and cells were suspended in $15 \mathrm{~mL}$ of PBS. $0.2 \mathrm{~mL}$ of GO-CS-Xn and GO-CS-Xn-DOX suspensions were treated with an equivalent volume of RBC suspension at concentrations of $20,40,60,80$ and $100 \mu \mathrm{g} / \mathrm{mL}$. The suspensions were shaken moderately and incubated for 30 min at $25^{\circ} \mathrm{C}$. After incubation, the resultant mixture was centrifuged at $1000 \mathrm{~g}$ for $10 \mathrm{~min}$, and the absorbance of the supernatant was estimated at 540nm with UV-Vis spectroscopy. RBC treated with $2 \%$ Triton X-100 served as a positive control, while RBC treated with PBS served as a negative control. The hemolysis percentage of RBCs was calculated using the formula: 


$$
\text { Hemolysis }(\%)=100 \times \frac{(\text { OD sample }- \text { OD PBS })}{(\text { OD Triton - OD PBS })}
$$

The effect of GO-CS-Xn and GO-CS-Xn-DOX on the RBC was investigated with optical microscopy.

\subsection{In vitro release of $D O X$.}

The DOX-loaded nanocarrier was resuspended into PBS pH 7.4 (the physiological $\mathrm{pH}$ ) and into $\mathrm{pH} 5.3$ (the $\mathrm{pH}$ of cancer cells) incubated at $37^{\circ} \mathrm{C}$. To maintain the volume constant, $3 \mathrm{~mL}$ of the solution was withdrawn from the reaction mixture at regular intervals, followed by the addition of freshly prepared PBS. The concentration of DOX released by GO-CS-Xn was determined using UV-Vis spectroscopy at 480nm.

\subsection{Kinetic model for DOX release.}

To investigate the drug release mechanism of DOX from GO-CS-Xn, the obtained drug release data were processed and fitted into the following kinetic models: zero-order, first-order, Higuchi model, and Korsmeyer-Peppas model [20].

\subsection{Cell toxicity studies.}

The in vitro cytotoxicity of prepared samples was studied on human glioblastoma cancer cells, U 87 by MTT assay.

\subsubsection{MTT cytotoxicity assay.}

The cytotoxic assessment of free DOX, GO-DOX, GO-CS-DOX and GO-CS-Xn-DOX was observed with 3-(4, 5-dimethylthiazol-2-yl)-2, 5-diphenyltetrazolium bromide (MTT assay). A cell count of $1 \times 10^{5} \mathrm{U} 87$ cells/well was seeded into a 96-well plate in DMEM by incubating at $37^{\circ} \mathrm{C}$ under $5 \% \mathrm{CO}_{2}$ and $95 \%$ air for $24 \mathrm{~h}$. After $24 \mathrm{~h}$, the plates were washed with 1001 of PBS and then treated with concentrations ranging $(20-100 \mathrm{~g} / \mathrm{mL})$ of free DOX, GODOX, GO-CS-DOX, and GO-CS-Xn-DOX to assess the cytotoxicity of graphene-based nanomaterials. After incubation, the U87 cells were treated with MTT $(5 \mathrm{mg} / \mathrm{ml}, 1001)$ in each well and incubated for another 4 hours. The formazan crystals formed in the cells were replaced with $100 \mu \mathrm{l}$ of dimethyl sulphoxide (DMSO). A microplate reader was used to measure absorbance at $570 \mathrm{~nm}$.

\subsection{Statistical Analysis.}

SPSS software version 16.0 was used for statistical analysis. It included one-way analysis of variance (ANOVA) followed by Tukey multiple range tests, with statistical significance tested at $5 \%(\mathrm{P}<0.05)$ levels. The results are presented as the mean values $\pm \mathrm{SD}$.

\section{Results and Discussion}

\subsection{UV-Vis Spectroscopy.}

UV visible spectrum of GO shows a characteristic peak at $229 \mathrm{~nm}$ ascribed to $\pi-\pi^{*}$ transitions of aromatic $-\mathrm{C}=\mathrm{C}$ - bonds $[50,51]$. After functionalization with $\mathrm{CS}$ and further conjugation with $\mathrm{Xn}$, the peak shifted to $278 \mathrm{~nm}$. DOX loading was found to have a strong 
absorbance band at $232 \mathrm{~nm}, 288 \mathrm{~nm}$, and $480 \mathrm{~nm}$, indicating a strong interaction between the DOX and the nanocarrier system GO-CS-Xn nanoparticles (Figure 1).

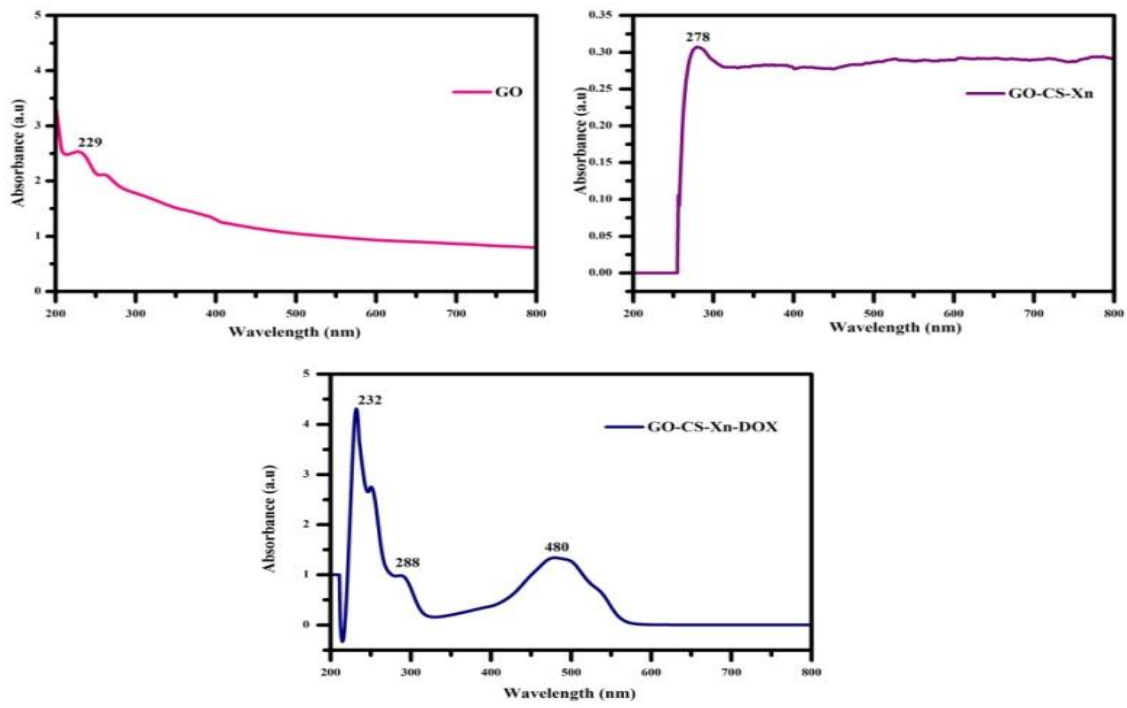

Figure 1. UV-Vis Spectra of GO, GO-CS-Xn, and GO-CS-Xn-DOX.

\subsection{FT-IR Spectroscopy.}

The functional group interactions of GO, GO-CS-Xn, and GO-CS-Xn-DOX were confirmed by FT-IR analysis (Figure 2). In the spectrum of GO we observed an abroad absorption band at $3424 \mathrm{~cm}^{-1}$ related to the hydroxyl group. The absorption bands at $1740 \mathrm{~cm}^{-}$ $1,1638 \mathrm{~cm}-1$ and $1402 \mathrm{~cm}^{-1}$ ascribed to the $\mathrm{C}=\mathrm{O}$ (carboxyl/carbonyl groups), $\mathrm{C}=\mathrm{C}$ (aromatics) and $\mathrm{C}-\mathrm{O}$ (carboxyl group. The weak band at $1207 \mathrm{~cm}^{-1}$ and two sharp peaks at $1103 \mathrm{~cm}^{-1}$ and $1029 \mathrm{~cm}^{-1}$ were due to the $\mathrm{C}-\mathrm{OH}$ stretching and the vibrations of epoxy (C-O-C) groups present at the edges of the GO planes. These GO findings revealed that many hydroxyl, carboxyl, and epoxy groups were present on the surface of GO[52]. Moreover, in the spectra of GO-CS-Xn, the peaks at $3433 \mathrm{~cm}^{-1}, 1632 \mathrm{~cm}^{-1}, 1389 \mathrm{~cm}^{-1}$ correspond to the GO peaks, peak at $2927 \mathrm{~cm}^{-1}$ and $2820 \mathrm{~cm}^{-1}$ relate to the $-\mathrm{CH}_{2}$ extending vibrations, and dominant peaks were observed at $1080 \mathrm{~cm}^{-1}$ and $1571 \mathrm{~cm}^{-1}$ ascribed to the absorbance of glycosidic bond and stretching vibration of $\mathrm{C}=\mathrm{O}$ of $-\mathrm{NHCO}$ - and $\mathrm{N}-\mathrm{H}$ bending of $\mathrm{NH}_{2}$. The FT-IR spectra of GO-CS-Xn-DOX show the characteristic peaks of DOX and the nanocarrier (GO-CS-Xn), thus confirming DOX's effective loading onto GO-CS-Xn.

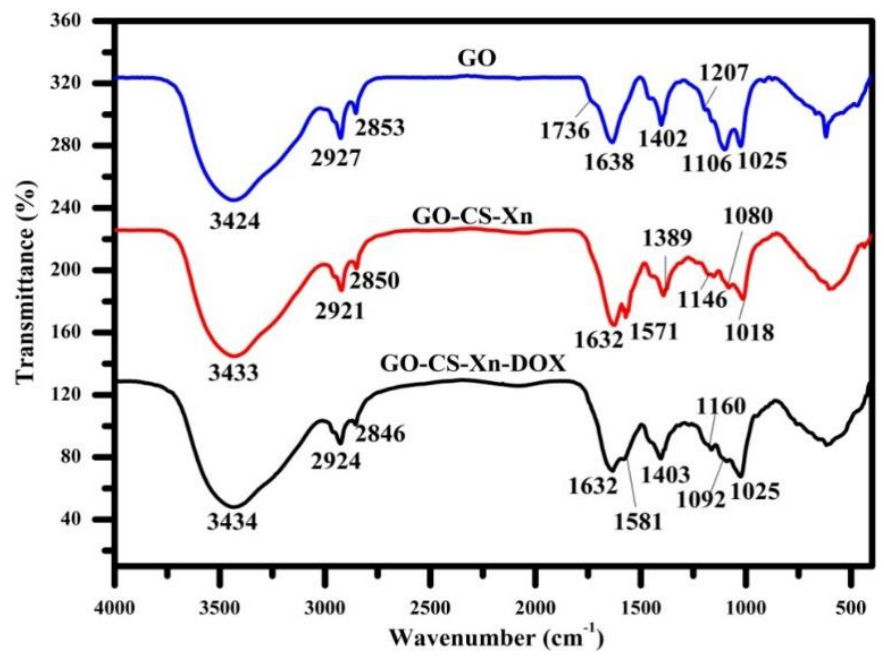

Figure 2. FT-IR Spectrum of GO, GO-CS-Xn, and GO-CS-Xn-DOX. 


\subsection{XRD Spectroscopy.}

XRD pattern of GO (Figure 3) exhibits a strong and sharp diffraction peak at $10.8^{\circ}$ corresponds to the 001 plane, represented a layer to layer distance of $0.82 \mathrm{~nm}$ (d-spacing). The increased interlayer spacing could be attributed to intercalation and the bonding effect of oxygen-containing functional groups. In addition, a diffraction peak was observed at 42.9, correspondings to the 101 plane, with a d-spacing of $0.210 \mathrm{~nm}$. Conjugation of CS and Xn on to GO (Figure 3) can be clearly observed with the two sharp peaks at $24.4^{\circ}(\mathrm{d}=0.365 \mathrm{~nm})$ and $43.3^{\circ}(\mathrm{d}=0.208 \mathrm{~nm})$ and two distorted peaks at $26.8^{\circ}(\mathrm{d}=0.329 \mathrm{~nm})$ and $36.1^{\circ}(\mathrm{d}=0.250 \mathrm{~nm})$. $\mathrm{XRD}$ results illustrate the formation of GO and its conjugation within the polymer matrix.

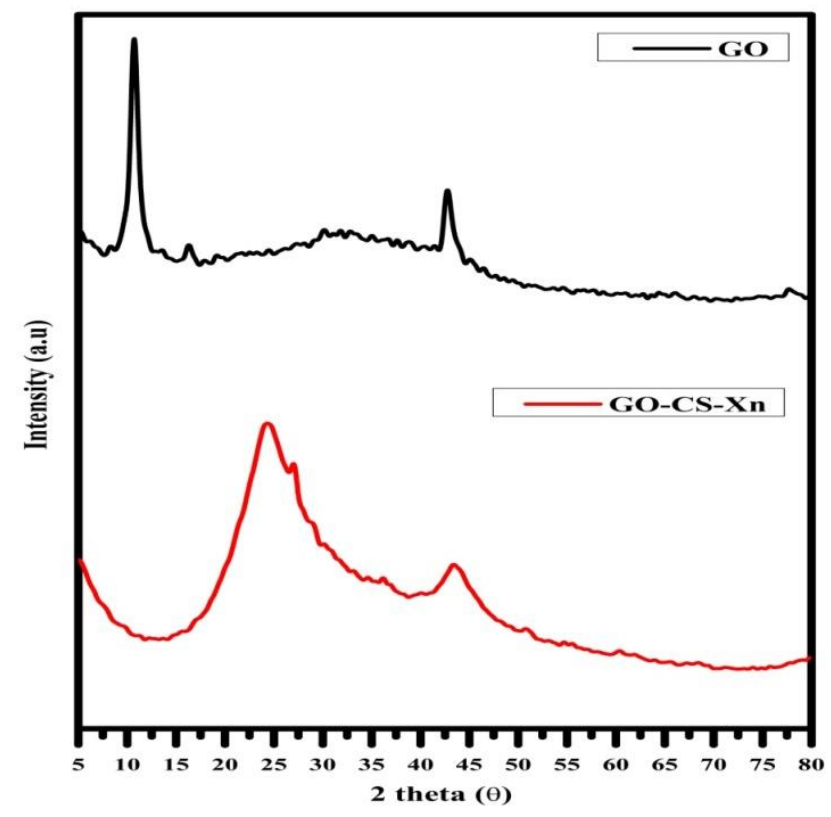

Figure 3. XRD spectra of GO and GO-CS-Xn.

\subsection{Raman Spectroscopy.}

Raman spectroscopy was used to investigate the carbon structure of GO and GO-CS$\mathrm{Xn}$, as shown in (Figure 4). The D and $\mathrm{G}$ bands seen at $1318 \mathrm{~cm}^{-1}$ and $1593 \mathrm{~cm}^{-1}$ are GO's characteristic peaks. The $\mathrm{G}$ band corresponds to the first-order scattering of the E2 $\mathrm{g}$ mode of the $\mathrm{sp}^{2}$ bonded carbon domains, whereas the $\mathrm{D}$ band at $1318 \mathrm{~cm}-1$ corresponds to the breathing method of A1g symmetry due to the existence of dangling bonds, which results in disordered lattice and disordered carbon domains, as well as other imperfections [53]. In addition to GOCS-Xn, there is a shift in the G and D bands at $1598 \mathrm{~cm}^{-1}$ and $1325 \mathrm{~cm}^{-1}$, respectively confirms the bonding interactions between the polymer and GO. The $\mathrm{G}$ band moved by $5 \mathrm{~cm}^{-1}$, and the $\mathrm{D}$ band moved by $7 \mathrm{~cm}^{-1}$ indicating a shift after functionalization. The intensity ratio $\left(\mathrm{I}_{\mathrm{D}} / \mathrm{I}_{\mathrm{G}}\right)$ demonstrates the disorders in the GO layers because of the oxidation of the graphite. $\mathrm{ID}_{\mathrm{D}} / \mathrm{IG}_{\mathrm{G}}$ proportion of GO is discovered to be 1.14 and for the GO-CS-Xn is 1.20 . When GO was functionalized by $\mathrm{CS}$ and $\mathrm{Xn}, \mathrm{I}_{\mathrm{D}} / \mathrm{I}_{\mathrm{G}}$ ratio increased from 1.14 to 1.20 , indicating the attachment of $\mathrm{CS} / \mathrm{Xn}$ onto the surface of GO altered the in-plane $\mathrm{sp}^{2}$ graphitic domains of $\mathrm{GO}$ and confirmed the functionalization of GO with $\mathrm{CS} / \mathrm{Xn}[6]$. 


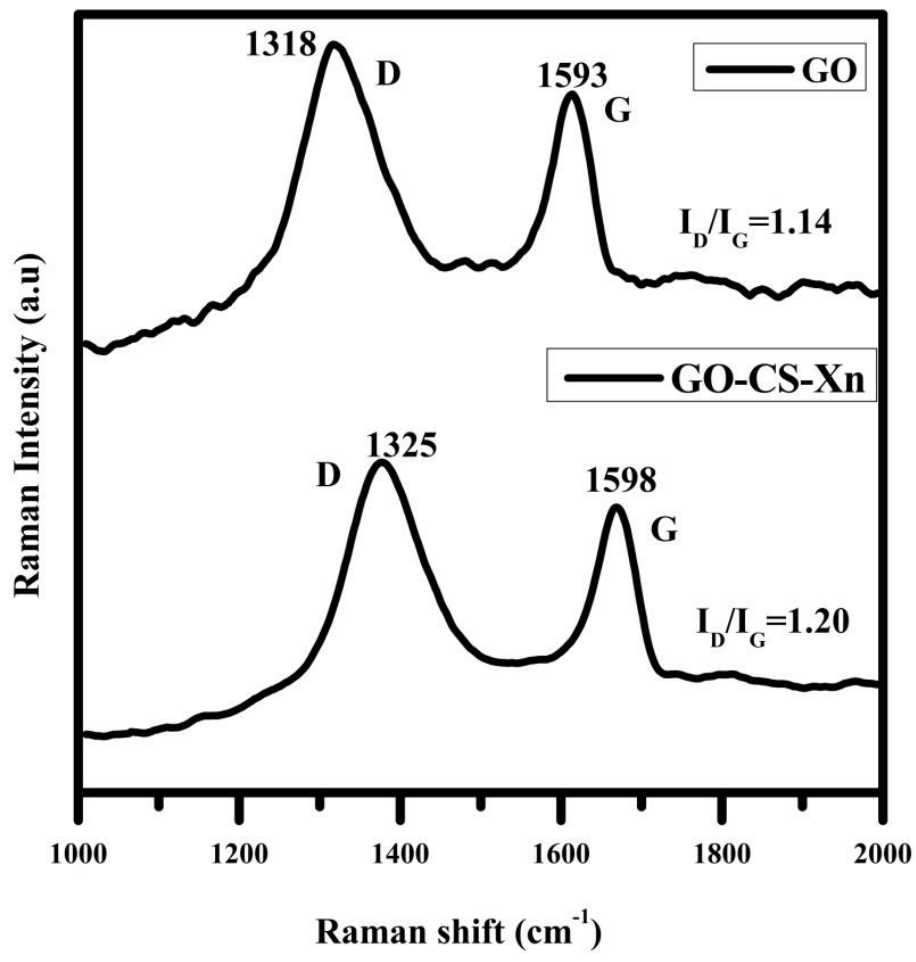

Figure 4.FT - Raman spectra of GO and GO-CS-Xn.

\subsection{Morphological analysis by SEM and TEM.}

The morphology of the prepared samples was demonstrated by the SEM and TEM images. SEM image of GO exhibits stacked flakes closely associate with each other, whereas after conjugation with CS and Xn, GO-CS-Xn shows porous surface, and, in addition, GO-CSXn-DOX depicts the embedding of DOX onto the prepared nanocarrier leads to the agglomeration of nanoparticles, thus indicating a change in the surface morphology (Figure 5).
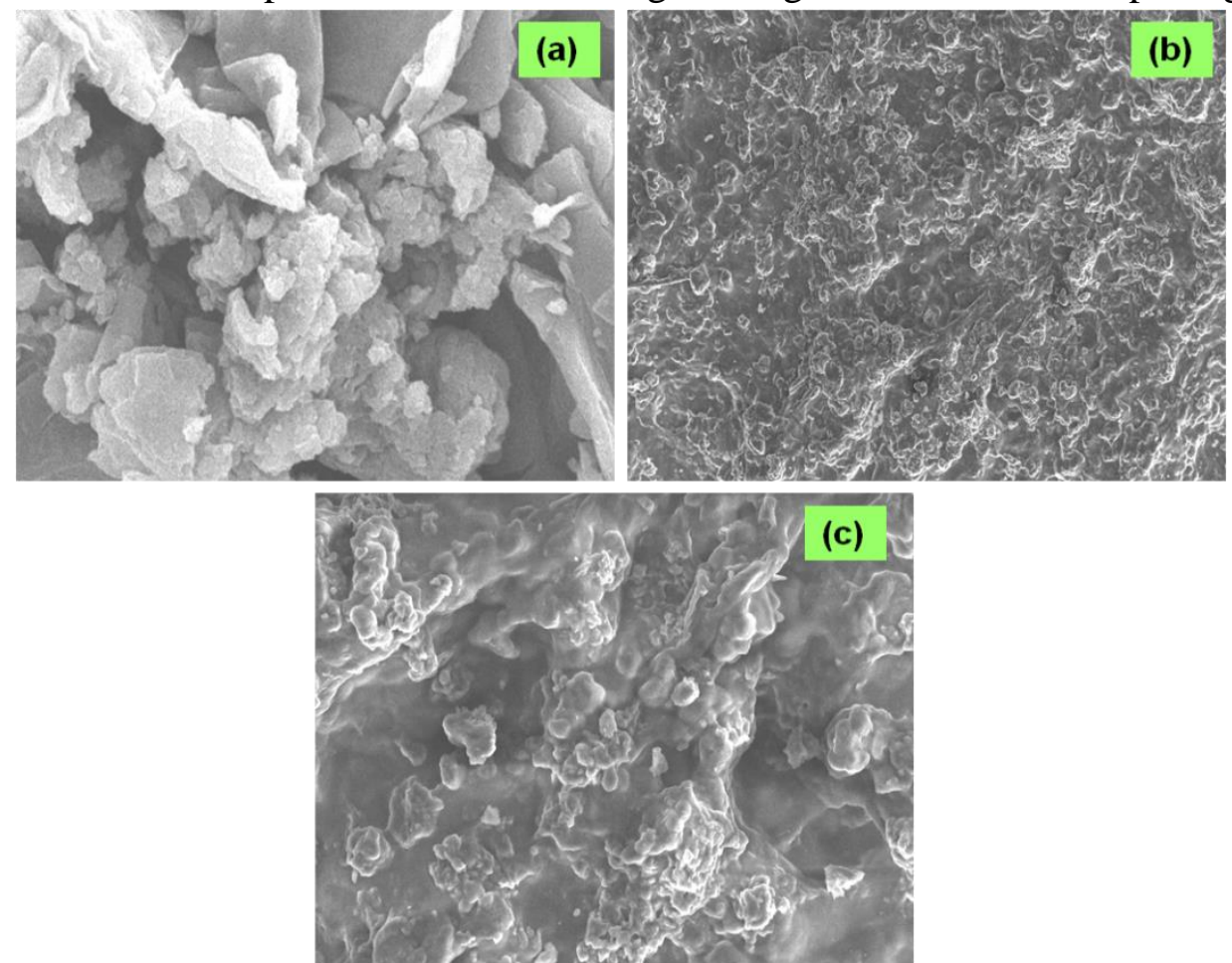

Figure 5. SEM images of (a) GO; (b) GO-CS-Xn; (c) GO-CS-Xn-DOX. 
TEM image of GO shows a layered structure of flat and transparent-like appearance with wrinkles on the surface. TEM image of GO-CS-Xn and GO-CS-Xn-DOX indicates an adhesion between DOX and GO-CS-Xn. From the TEM analysis, it is clear that this nanocarrier system GO-CS-Xn brings an intrinsic change upon conjugation and loading of DOX (Figure 6).

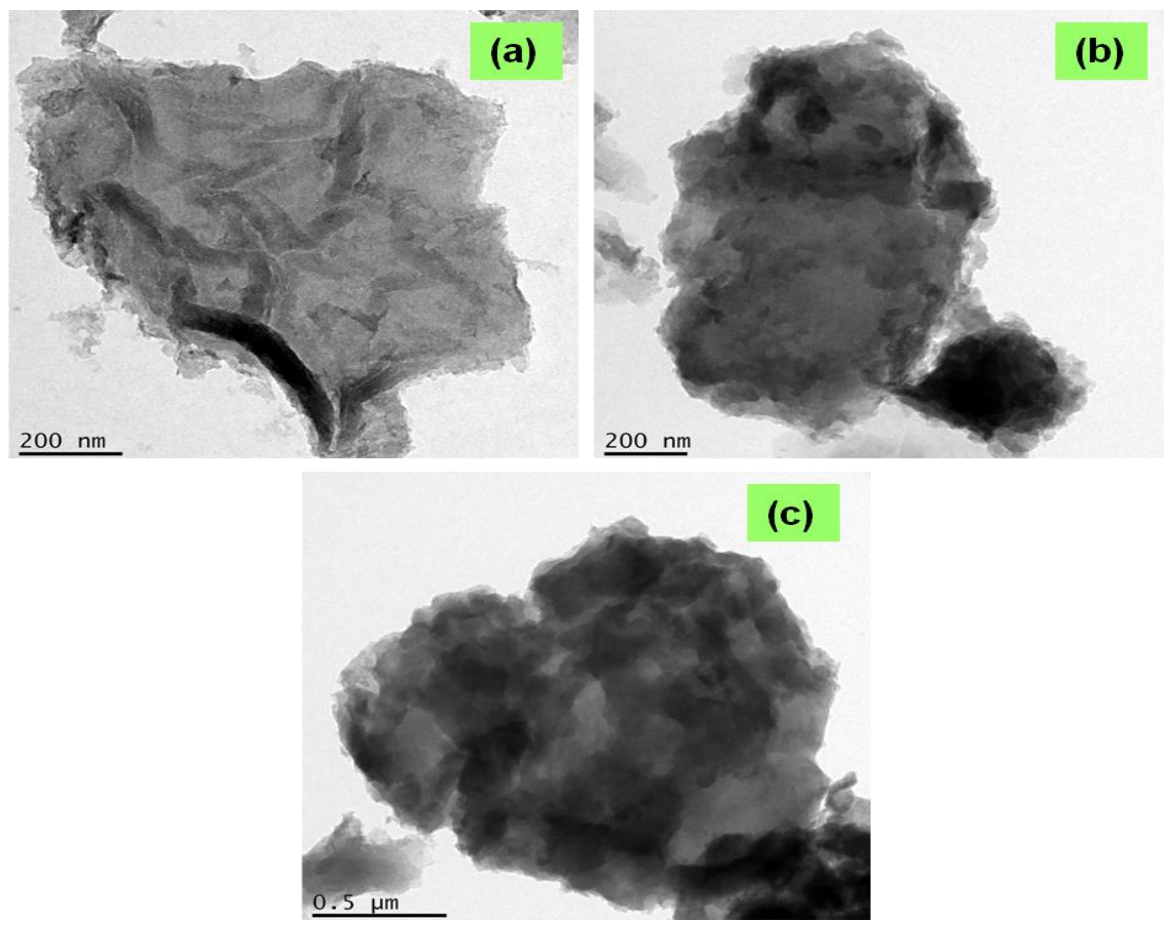

Figure 6. TEM images of (a) GO; (b) GO-CS-Xn; (c) GO-CS-Xn-DOX.

\subsection{Zeta potential and particle size distribution.}

Colloidal stability of GO is crucial for controlling the performance of drug carriers as an excellent candidate for graphene-based biomaterial [54]. Table 1 shows the hydrodynamic sizes of GO, GO-CS, and GO-CS-Xn in distilled water as determined by Zetasizer. The mean size of GO was $132.911 .70 \mathrm{~nm}$ before conjugation with CS, and after conjugation with CS, the GO-CS becomes (144.9 30.6nm) and GO-CS-Xn (271.8 9nm). This change in the size confirms the successful attachment of CS/Xn onto the GO surface.

The surface charge and the stability of aggregated nanoparticles were observed by Zeta potential measurements (Table 1). The surface charge of prepared GO $(-24.4 \pm 1.6 \mathrm{mV})$, GOCS $(+25.6 \pm 0.5 \mathrm{mV})$, GO-CS-Xn $(-20.4 \pm 0.8 \mathrm{mV})$ and GO-CS-Xn-DOX $(-19.8 \pm 1.2 \mathrm{mV})$. The changes in zeta potential values indicate that GO was successfully modified with CS and Xn and has the potential for good biocompatibility. GO-CS-Xn-DOX is less negative compared to GO.

Table1. Zeta potential values, mean particle size, and polydispersity index of GO before and after surface modification.

\begin{tabular}{l|c|c|c} 
Nanocarrier & $\begin{array}{l}\text { Zeta potential } \\
(\mathbf{m V})\end{array}$ & $\begin{array}{c}\text { Mean particle } \\
\text { size(nm) }\end{array}$ & $\begin{array}{c}\text { Polydispersity } \\
\text { index }\end{array}$ \\
\hline GO & $-24.4 \pm 1.6$ & $132.9 \pm 11.70$ & 0.449 \\
GO-CS & $+25.6 \pm 0.5$ & $144.9 \pm 30.6$ & 0.377 \\
GO-CS-Xn & $-20.4 \pm 0.8$ & $271.8 \pm 9$ & 0.234
\end{tabular}


This is most likely due to the ionization of the carboxylate/ carboxylic group, as well as the coating of CS and Xn on the GO surface. As a result, these groups are responsible for the formation of negative charges following DOX loading.3.7. Hemolysis assay.

In vitro hemocompatibility analysis plays a prominent role in the field of biomedical applications. For intravenous injection and drug delivery, the nanocarrier should have great blood compatibility. The RBC membranes were incubated with different concentrations of GOCS-Xn and GO-CS-Xn-DOX. At a concentration of $100 \mu \mathrm{g} / \mathrm{mL}$; GO-CS-Xn exhibits a hemolytic effect of 10\%, whereas after loading of DOX onto GO-CS-Xn, which brings about a reasonable change in RBCs toxicity of about $1.4 \%$ at the same concentration (Figure $7 \mathrm{a}$ and $7 b$ ), indicating a low or no toxicity in RBCs makes this nanocarrier more biocompatible and better suited for drug delivery applications. Optical microscopy images of RBCs were observed and shown in Figure 7c.

(a)
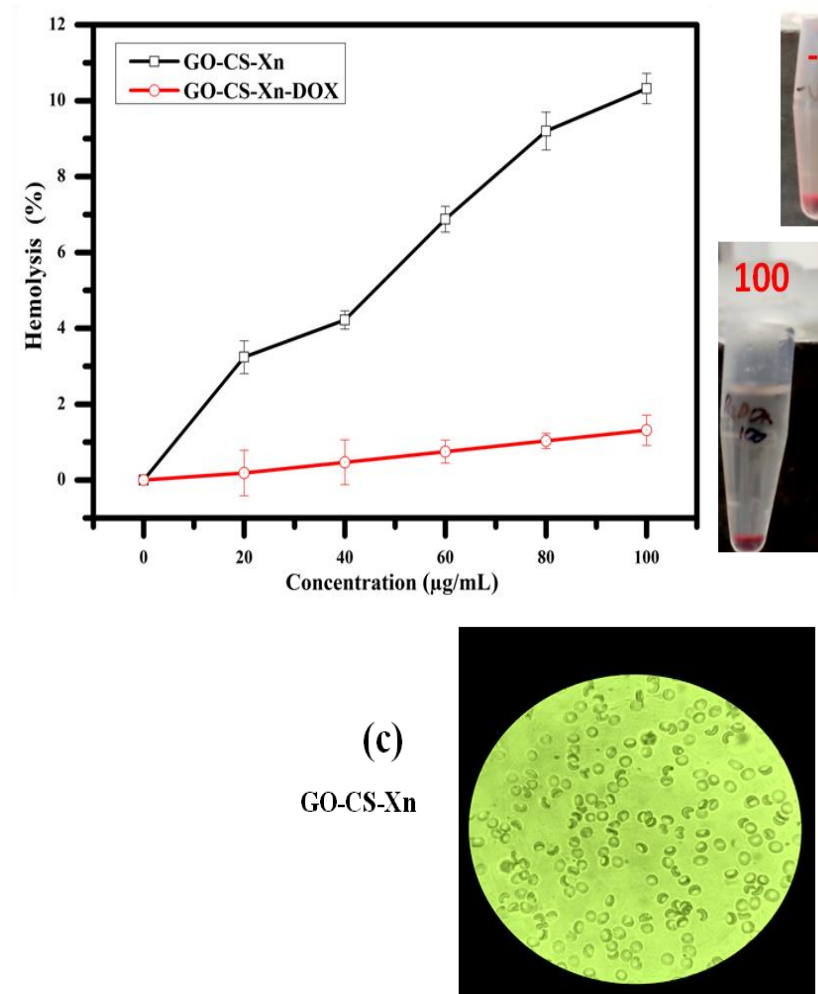

(b)

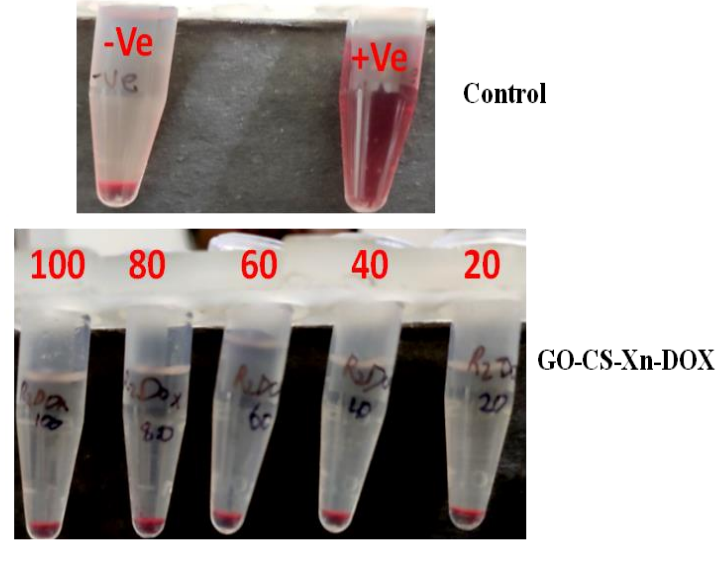

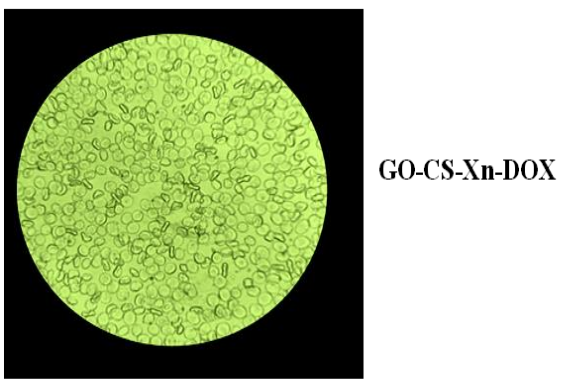

Figure 7. (a) Hemolytic activity of GO-CS-Xn and GO-CS-Xn-DOX; (b) Visually observe the presence of hemoglobin in the supernatant; (c) optical microscopy images of RBC s with GO-CS-Xn and GO-CS-Xn DOX.

\subsection{Loading efficiency of DOX.}

Table 2 shows the percentage of DOX loaded onto GO and GO-CS-Xn - stacking and hydrophobic interactions between GO and DOX using a simple mixture and sonication method. Centrifugation was used to remove the unbound drug, and the loading efficiency of DOX onto the functionalized GO-based nanocarrier was calculated by measuring the concentration of the unbound drug-using UV-Vis spectra. According to the data in (Table 2), GO-CS-Xn's loading efficiency and loading capacity are as high as $73.5 \%$ and $81.8 \%$, respectively, when compared to GO. Apart from these, some surface areas of GO are already occupied by amino polysaccharide CS and Xn polymer, resulting in high drug loading and efficiency compared with the GO. 
Table 2. Loading capacity and loading efficiency of DOX loaded onto GO and GO-CS-Xn.

\begin{tabular}{l|c|c} 
Nanocarrier & \%LC & \%LE \\
\hline GO & 48.0 & 44.5 \\
GO-CS-Xn & 81.8 & 73.5
\end{tabular}

\subsection{In vitro release of $D O X$}

In order to evaluate the potential use of GO-CS-Xn as a nanocarrier for drug delivery systems, the in vitroDOX release studies were carried out under different $\mathrm{pH}$, including endosomal $\mathrm{pH}$ of cancer cells (5.3) and physiological $\mathrm{pH}$ (7.4). Figure 8 depicts the release behavior of DOX from GO-CS-Xn over a 70-h period. As observed from Figure 8, at normal physiological conditions ( $\mathrm{pH} 7.4$ ), the release of DOX from GO-CS-Xn nanocarrier is slow and only $28 \%$ of the total bound DOX is released after $70 \mathrm{~h}$, which may be due to the strong hydrogen bonding effect between DOX and nanocarrier leads to slow release. In acidic conditions ( $\mathrm{pH}$ 5.3), the rapid release of DOX is enhanced by $70 \%$ in $70 \mathrm{~h}$, which may be due to the protonation of amine groups and the breaking of hydrogen bonds between DOX and the nanocarrier.

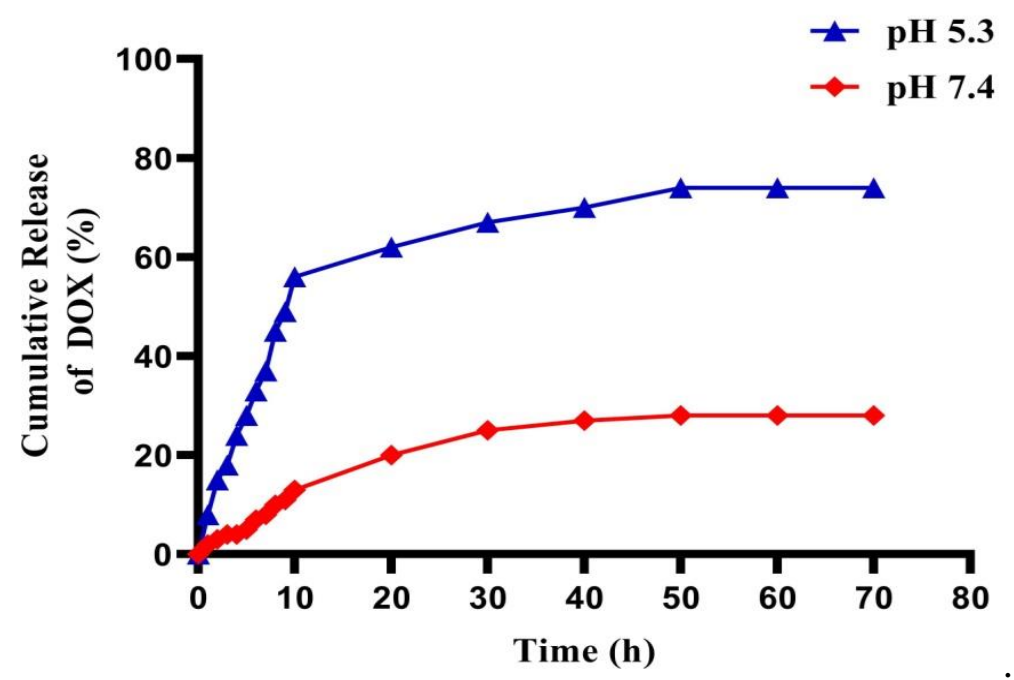

Figure 8. In vitro release of DOX from GO-CS-Xn carrier at $\mathrm{pH} 5.3$ and $\mathrm{pH} 7.4$.

\subsection{DOX release mechanism from GO-CS-Xn-DOX nanocarrier.}

DOX release mechanism was evaluated using GO-CS-Xn-DOX, and the first $60 \%$ of the accumulative drug release data at $\mathrm{pH} 5.3$ and 7.4 were fitted into various kinetic models such as Zero order, First order, Higuchi, and Korsmeyer-Peppas models, as shown in (Figure 9). Table 3 summarizes the correlation coefficients $R 2$ and the release exponent $n$. The R2 value was used to determine the model that fits the release, and the $\mathrm{n}$ gave insights into the mechanism. According to the different kinetic models, the in vitro release fit the Higuchi release model within an $\mathrm{R} 2$ of 0.9159 and 0.9288 for $\mathrm{pH} 7.4$ and $\mathrm{pH} 5.3$, respectively, indicating a simple diffusion DOX release.

However, apart from the correlation coefficient, the $\mathrm{n}$ value of the Korsmeyer Peppas model is also used to evaluate the DOX release mechanism. Accordingly, the DOX release mechanism at $\mathrm{pH} 7.4$ is case II transport $(\mathrm{n}=0.8362)$. At $\mathrm{pH} 5.3$ is anomalous or non-fickian diffusion $(\mathrm{n}=0.5407)$, which is correlated to a combination of drugs diffusion and polymer dissolution. [55, 56]. 
Table 3. Correlation coefficients and release exponents of DOX from GO-CS-Xn at pH 7.4 and pH 5.3 obtained from four kinds of kinetic models.

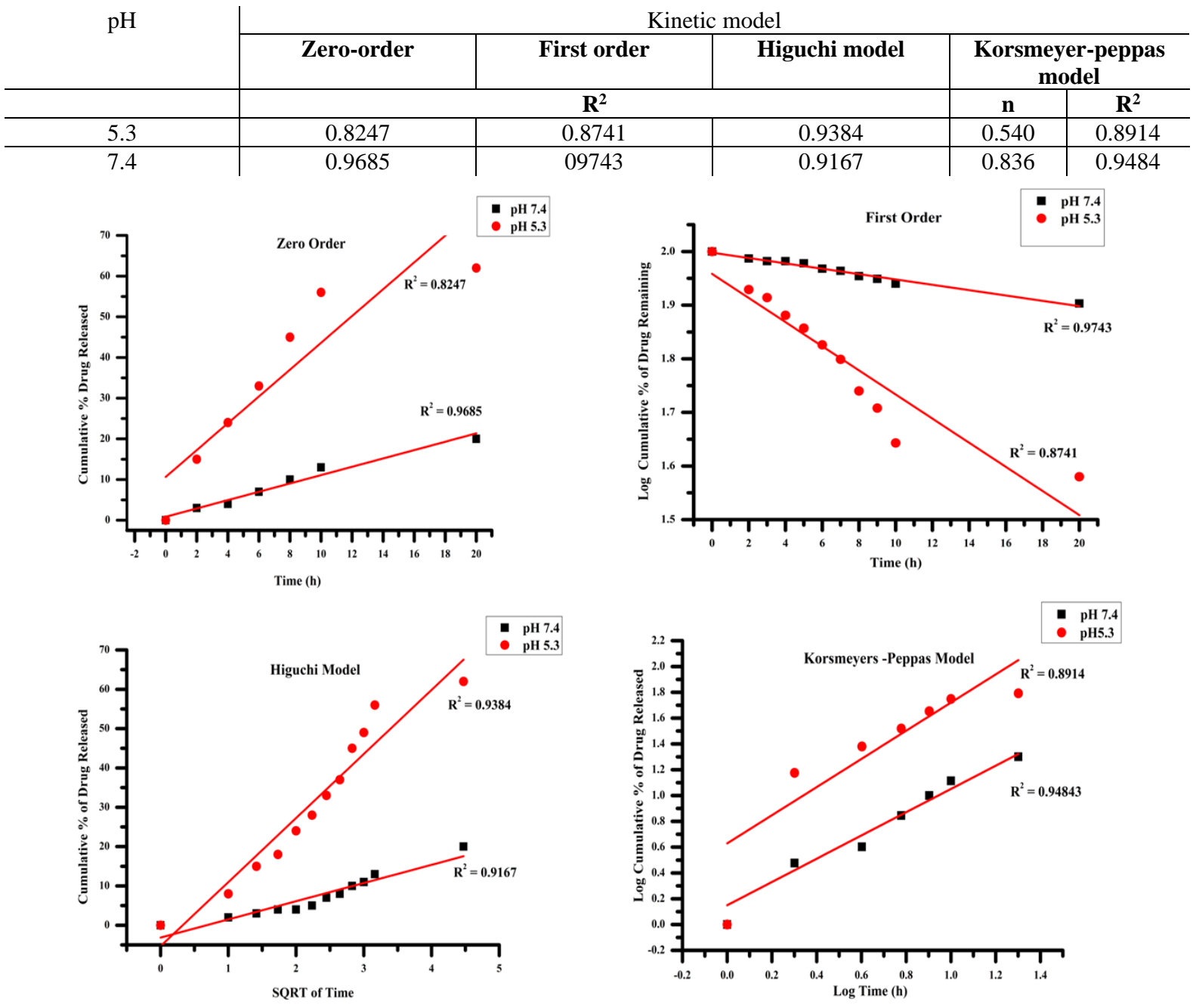

Figure 9. Kinetic models of the Zero order, First order, Higuchi and Korsmeyer-Peppas model of DOX release from GO-CS-Xn.

\subsection{In vitro cytotoxicity studies.}

To investigate the toxicity of DOX loaded on the GO-CS-Xn nanocarrier, cytotoxicity studies were performed by MTT assay using human glioblastoma U 87 tumor cells. DOX, GO, and GO-CS-Xn-DOX were incubated with U 87 cells for $24 \mathrm{~h}$. The study shows cell viability values as the percentage of viable cells in the treated sample to the untreated sample. As shown in (Figure 10) at a concentration of $100 \mathrm{~g} / \mathrm{mL}$, free DOX, GO-DOX, and GO-CS-Xn-DOX reduce cell growth by up to $61.55 \pm 0.76,76.56 \pm 0.90$, and $48 \pm 1.02 \%$ at a concentration of $100 \mu \mathrm{g} / \mathrm{mL}$, indicating that the GO-CS-Xn-DOX has the potential for selectively killing tumor cells in vitro and also indicating the excellent biocompatibility of the constituent bioactive polysaccharides. However, due to the inefficient release of DOX from the GO, GO-DOX has the highest toxicity to $\mathrm{U} 87$ cells at the same concentration. Morphological images of $\mathrm{U} 87$ cell lines treated with various concentrations of DOX, GO-DOX, and GO-CS-Xn-DOX were shown in Figure 11. 


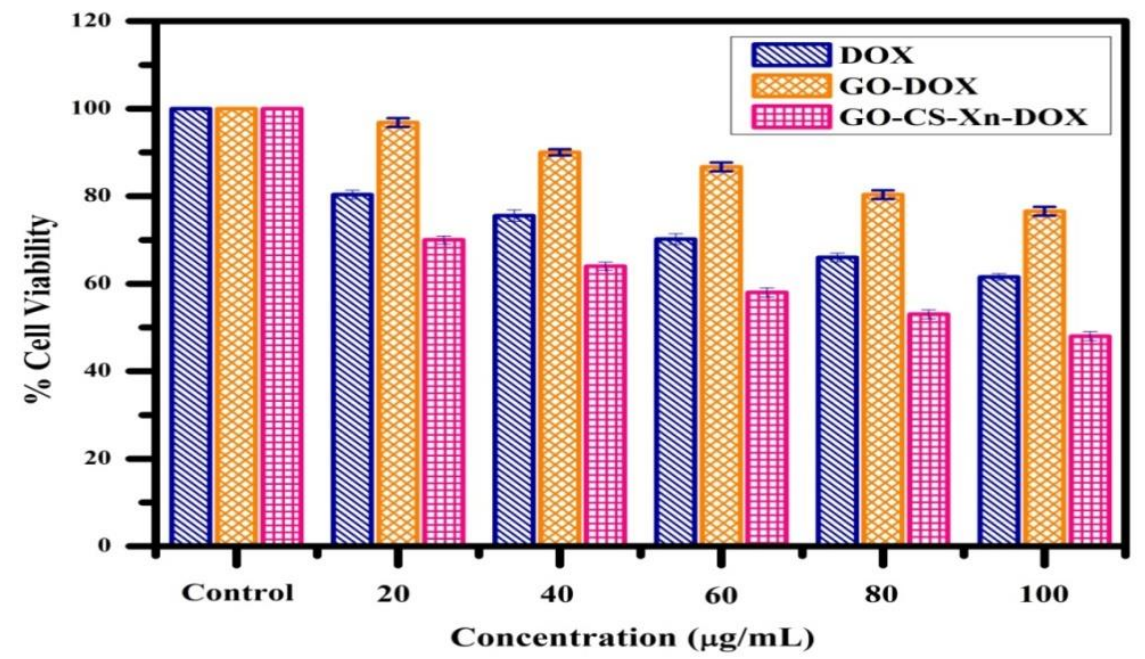

Figure 10.Cell viability of U 87 cells with different concentrations of DOX, GO-DOX, and GO-CS-Xn-DOX.
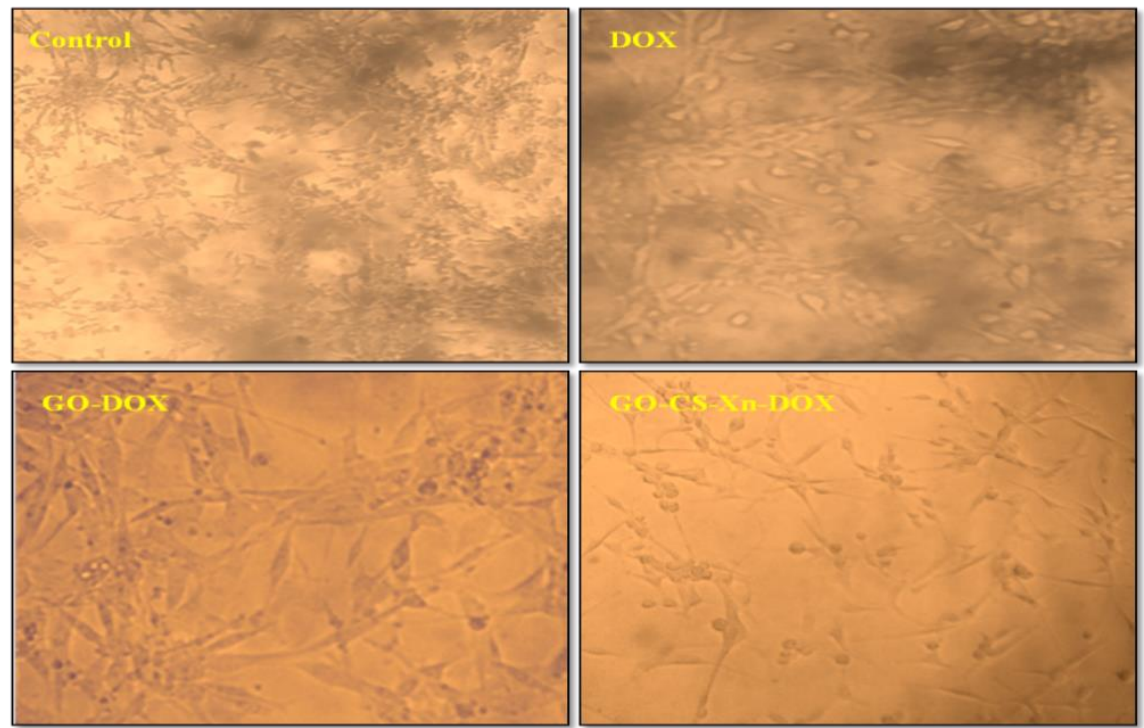

Figure 11.Morphological images of U 87 cells treated with Control, GO-CS, and GO-CS-Xn-DOX at 100 $\mu \mathrm{g} / \mathrm{ml}$ upon $24 \mathrm{~h}$ of incubation.

\section{Conclusions}

In this work, we attempted to develop a new nanocarrier based on graphene material to treat glioblastoma cancer. Initially, Graphene oxide was prepared by the Hummers method. GO nanoparticles were first coated with a biocompatible amino polysaccharide, CS, which provides an anchor for further conjugation. The bioactive tamarind polysaccharide Xn was successfully grafted onto GO-CS to improve biocompatibility and drug loading efficiency. The developed nanocarrier, GO-CS-Xn, was characterized by using techniques such as UV-Vis, FTIR, XRD, SEM, TEM, Raman spectroscopy, and zeta potential measurements to ensure the successful conjugation of $\mathrm{CS}$ and Xn onto the GO surface. The water-soluble anti-tumor model drug DOX was chosen for loading onto GO-CS-Xn nanocarrier. Studies on drug release were carried to explore the interaction of DOX in the physiological and cancerous cell environment. A high rate of drug loading and $\mathrm{pH}$-dependent release of DOX has been proved by fitting into various kinetic models, thereby following an anomalous diffusion and super case II mechanism. The biocompatibility of nanocarriers and drug-loaded nanocarriers was investigated using the hemolysis assay. The GO-CS-X-DOX system was tested for cytotoxicity 
against U 87 tumor cell line. It exhibited higher cytotoxicity of $51.7 \%$ against cell lines. The current study is a brief screening of bioactive polysaccharides used to synthesize a newer platform, GO-CS-Xn, for tumor drug delivery. Further research will be conducted to demonstrate the efficacy of drug delivery via nanocarrier in vivo.

\section{Funding}

This research received no external funding.

\section{Acknowledgments}

This research has no acknowledgment.

\section{Conflicts of Interest}

The authors confirm that this article has no conflict of interest.

\section{References}

1. Siegel, R.L.; Miller, K.D.; Jemal, A. Cancer statistics, 2019. CA: A Cancer Journal for Clinicians 2019, 69, 7-34, https://doi.org/10.3322/caac.21551.

2. Liu, B.; Che, C.; Liu, J.; Si, M.; Gong Z, Li, Y.; Yang, G. Fabrication and Antitumor Mechanism of a Nanoparticle Drug Delivery System: Graphene Oxide/Chitosan Oligosaccharide/ $\gamma$-Polyglutamic Acid Composites for Anticancer Drug Delivery. Chemistry Select 2019, 4, 12491-12502, https://doi.org/10.1002/slct.201903145.

3. Karthika, V.; AlSalhi, M.S.; Devanesan, S.; Gopinath, K.; Arumugam, A.; Govindarajan, M. Chitosan Overlaid $\mathrm{Fe}_{3} \mathrm{O}_{4} / \mathrm{rGO}$ Nanocomposite for Targeted Drug Delivery, Imaging, and Biomedical applications. Scientific Reports 2020, 3, 10, 18912, https://doi.org/10.1038/s41598- 020-76015-3.

4. Arias, J.L.; Reddy, L.H.; Couvreur, P. Fe3O4/ Chitosan Nanocomposite for Magnetic Drug Targeting to Cancer. Journal of Materials Chemistry 2012, 22, 7622-7632, https://doi.org/10.1039/C2JM15339D.

5. Zuchowska, A.; Chudy, M.; Dybko, A.; Brzozka. Z. Graphene as a new material in anticancer therapy-in vitrostudies. Sensors and Actuators B: Chemical 2017, 243, 152-165, https://doi.org/10.1016/j.snb.2016.11.105.

6. Borandeh, S.; Abdolmaleki, A.; Abolmaali, S.S.; Tamaddon, A.M. Synthesis, structural and in-vitro characterization of $\beta$-cyclodextrin grafted L-phenylalanine functionalized graphene oxide nanocomposite: A versatile nanocarrier for $\mathrm{pH}$-sensitive doxorubicin delivery. Carbohydrate Polymers 2018, 201,151-161, https://doi.org/10.1016/j.carbpol.2018.08.064.

7. Wakaskar, R.R. General overview of lipid-polymer hybrid nanoparticles, dendrimers, micelles, liposomes, spongosomes and cubosomes. Journal of Drug Targeting 2018, 26, 311-318, https://doi.org/10.1080/1061186X.2017.1367006.

8. Torres, J.; Liu, Y.; So, S.; Yi, H.; Park, S.; Lee, J.K.; Lim, S.C.; Yun, M. Effects of Surface Modifications to Single and Multilayer Graphene Temperature Coefficient of Resistance. ACS Appl. Mater. Interfaces 2020, 12, 48890-48898, https://doi.org/10.1021/acsami.0c09621.

9. Liu, W.; Speranza, G. Tuning the Oxygen Content of Reduced Graphene Oxide and Effects on Its Properties. ACS Omega 2021, 6, 6195-6205, https://doi.org/10.1021/acsomega.0c05578.

10. Patil, T.V.; Patel, D.K.; Dutta, S.D.; Ganguly, K.; Lim, K.T. Graphene Oxide-Based Stimuli-Responsive Platforms for Biomedical Applications. Molecules, 26, 2797, https://doi.org/10.3390/molecules26092797.

11. Javanbakht, S.; Pooresmaeil, M.; Namazi, H. Green one-pot synthesis of carboxymethylcellulose/Zn-based metal-organic framework/graphene oxide bio-nanocomposite as a nanocarrier for drug delivery system. Carbohydr. Polym. 2019, 208, 294-301, https://doi.org/10.1016/j.carbpol.2018.12.066.

12. Morales-Narvaez, E.; Merkoci, A. Graphene Oxide as an Optical Biosensing Platform: A Progress Report. Adv. Mater. 2019, 31, 1805043, https://doi.org/10.1002/adma.201805043. 
13. Yogesh, G.K.; Shuaib, E.P.; Roopmani, P.; Gumpu, M.B.; Krishnan, U.M.; Sastikumar, D. Synthesis, characterization and bioimaging application of laser-ablated graphene-oxide nanoparticles (nGOs) Diam. Relat. Mater. 2020, 104, 107733, https://doi.org/10.1016/j.diamond.2020.107733.

14. Martín, C.; Ruiz, A.; Keshavan, S.; Reina, G.; Murera, D.; Nishina, Y.; Fadeel, B.; Bianco, A. A Biodegradable Multifunctional Graphene Oxide Platform for Targeted Cancer Therapy. Adv. Funct. Mater. 2019, 29, 1901761, https://doi.org/10.1002/adfm.201901761.

15. Khalili, R.; Zarrintaj, P.; Jafari, S.H.; Vahabi, H.; Saeb, M.R. Electroactive poly (p-phenylene sulfide)/rgraphene oxide/chitosan as a novel potential candidate for tissue engineering. Int. J. Biol. Macromol. 2020, 154, 18-24, https://doi.org/10.1016/j.ijbiomac.2020.03.029.

16. Ahmed, M.K.; Mansou,r S.F.; Al-Wafi, R.; Menazea, A.A. Composition and design of nanofibrous scaffolds of $\mathrm{Mg} / \mathrm{Se}$ - hydroxyapatite/graphene oxide@ @-polycaprolactone for wound healing applications. J. Mater. Res. Technol. 2020, 9, 7472-7485, https://doi.org/10.1016/j.jmrt.2020.04.094.

17. Anirudhan, T.S.; Chithra Sekhar, V.; Athira, V.S. Graphene oxide based functionalized chitosan polyelectrolyte nanocomposite for targeted and $\mathrm{pH}$ responsive drug delivery. Int J Biol Macromol. 2020, 150, 468-479, https://doi.org/10.1016/j.ijbiomac.2020.02.053.

18. Jafari, Z.; Rad, A.S.; Baharfar, R.; Asghari, S.; Esfahani, MR. Synthesis and application of chitosan/tripolyphosphate/graphene oxide hydrogel as a new drug delivery system for Sumatriptan Succinate. J. Mol. Liq. 2020, 315, 113835, https://doi.org/10.1016/j.molliq.2020.113835.

19. Abdelhamid, H.N.; Hussein,K.H. Graphene Oxide as a Carrier for Drug Delivery of Methotrexate. Biointerface Research in Applied Chemistry 2021, 11, 14726-14735, https://doi.org/10.33263/BRIAC116.1472614735.

20. Tiwari, H.; Karki, N.; Pal, M.; Basak, S.; Verma, R.K.; Bal, R.; Kandpal, N.D.; Bisht, G.; Sahoo, NG. Functionalized graphene oxide as a nanocarrier for dual drug delivery applications: The synergistic effect of quercetin and gefitinib against ovarian cancer cells. Colloids Surf B Biointerfaces 2019 178, 452-459, https://doi.org/10.1016/j.colsurfb.2019.03.037.

21. Figueroa, T.; Aguayo, C.; Fernández, K. Design and Characterization of Chitosan-Graphene Oxide Nanocomposites for the Delivery of Proanthocyanidins. International journal of nanomedicine $\mathbf{2 0 2 0}$, 15, 1229-1238, https://doi.org/10.2147/IJN.S240305.

22. Pei, X.; Zhu, Z.; Gan, Z.; Chen, J.; Zhang, X.; Cheng, X.; Wan, Q.;Wang, J. PEGylated nano-graphene oxide as a nanocarrier for delivering mixed anticancer drugs to improve anticancer activity. Sci zzxRep 2020, 10, 2717, https://doi.org/10.1038/s41598-020-59624-w.

23. Dhanavel, S.; Revathy, T.A.; Sivaranjani, T.; Sivakumar, P.;Palani, V.; Narayanan, A. 5-Fluorouracil and curcumin co-encapsulated chitosan/reduced graphene oxide nanocomposites against human colon cancer cell lines. Polym. Bull. 2020, 77, 213-233, https://doi.org/10.1007/s00289-019-02734-x.

24. Pan, H.; Yu, Y.; Li, L.; Liu, B.; Liu, Y. Fabrication and Characterization of Taurine Functionalized Graphene Oxide with 5-Fluorouracil as Anticancer Drug Delivery Systems. Nanoscale Res Lett 2021, 16, 84, https://doi.org/10.1186/s11671-021-03541-y.

25. Vinothini, K.; Rajendran, N.K.; Rajan, M.; Ramu, A.; Marraiki, N.; Elgorban, A.M. A magnetic nanoparticle functionalized reduced graphene oxide-based drug carrier system for a chemo-photodynamic cancer therapy. N J Chem. 2020, 44, 5265-5277, https://doi.org/10.1039/D0NJ00049C.

26. Zaboli, M.; Raissi, H.; Moghaddam, N.R.; Farzad, F. Probing the adsorption and release mechanisms of cytarabine anticancer drug on/from dopamine functionalized graphene oxide as a highly efficient drug delivery system. J. Mol. Liq. 2020, 301, 112458, https://doi.org/10.1016/j.molliq.2020.112458.

27. Makvandi, P.; Ghomi, M.; Ashrafizadeh, M.; Tafazoli, A.; Agarwal, T.; Delfi, M.; Akhtari, J.; Zare, E.N.; Padil, V.V.T.; Zarrabi, A.; Pourreza, N.; Miltyk, W.; Maiti, T.K. A review on advances in graphenederivative / polysaccharide bionanocomposites: Therapeutics, pharmacogenomics and toxicity. Carbohydrate Polymers 2020, 250, 116952, https://doi.org/10.1016/j.carbpol.2020.116952.

28. Tolvanen, J.; Kilpijarvi, J.; Pitkanen, O.; Hannu, J.; Jantunen, H. Stretchable Sensors with Tunability and Single Stimuli-Responsiveness through Resistivity Switching Under Compressive Stress. ACS Appl. Mater. Interfaces 2020, 12, 14433-14442, https://doi.org/10.1021/acsami.0c00023.

29. Song, Y.-Y.; Liu, Y.; Jiang, H.-B.; Xue, J.-Z.; Yu, Z.-P.; Li, S.-Y.; Han, Z.-W.; Ren, L.-Q. Janus Soft Actuators with On-Off Switchable Behaviors for Controllable Manipulation Driven by Oil.ACS Appl. Mater. Interfaces 2019, 11, 13742-13751,https://doi.org/10.1021/acsami.8b20061. 
30. Abu-Thabit, N.Y.; Hamdy, A.S. Stimuli-responsive Polyelectrolyte Multilayers for fabrication of self-healing coatings-A review. Surface and Coatings Technology 2016, 303, 406-424, https://doi.org/10.1016/j.surfcoat.2015.11.020.

31. Chatterje,e S.; Chi-leung Hui, P. Review of Stimuli-Responsive Polymers in Drug Delivery and Textile Application. Molecules 2019, 24, 2547, https://doi.org/10.3390/molecules24142547.

32. Shu, T.; Hu, L.; Shen, Q.; Jiang, L.; Zhang, Q.; Serpe, M.J. Stimuli-responsive polymer-based systems for diagnostic applications. J. Mater. Chem. B. 2020, 8, 7042-7061, https://doi.org/10.1039/D0TB00570C.

33. Son, H.; Yoon, C. Advances in Stimuli-Responsive Soft Robots with Integrated Hybrid Materials. Actuators 2020, 9,115 , https://doi.org/10.3390/act9040115.

34. Li, Z.; Yin, Y. Stimuli-Responsive Optical Nanomaterials. Adv. Mater. 2019, 31, 1807061, https://doi.org/10.1002/adma.201807061.

35. Motiei, M.; Kashanian, S.; Lucia, L.A.; Khazaei, M. Intrinsic parameters for the synthesis and tuned properties of amphiphilic chitosan drug delivery nanocarriers. Journal of Controlled Release 2017, 260, $213-$ 225, https://doi.org/10.1016/j.jconrel.2017.06.010.

36. Di Martino, A.; Sedlarik, V. Amphiphilic chitosan-grafted-functionalized polylactic acid based nanoparticles as a delivery system for doxorubicin and temozolomide co-therapy. International Journal of Pharmaceutics2014, 474, 134-145, https://doi.org/10.1016/j.ijpharm.2014.08.014.

37. Di Martino, A.; Kucharczyk, P.; Zednik, J.; Sedlarik, V. Chitosan grafted low molecular weight polylactic acid for protein encapsulation and burst effect reduction. International Journal of Pharmaceutics 2015, 496, 912-921, https://doi.org/10.1016/j.ijpharm.2015.10.017.

38. Tao, F.; Cheng, Y.; Shi, X.; Zheng, H.; Du, Y.; Xiang, W.; Deng, H. Applications of chitin andchitosan nanofibers in bone regenerative engineering. Carbohydr. Polym. 2020, 230, 115658, https://doi.org/10.1016/j.carbpol.2019.115658.

39. Moeini, A.; Pedram, P.; Makvandi, P.; Malinconico, M.; d'Ayala, G. Wound healing and antimicrobial effect of active secondary metabolites in chitosan-based wound dressings: A review. Carbohydr. Polym. 2020, 233, 115839, https://doi.org/10.1016/j.carbpol.2020.115839.

40. Diop, M.; Auberval, N.; Viciglio, A.; Langlois, A.; Bietiger, W.; Mura, C.; Peronet, C.; Bekel, A.; Julien David, D.; Zhao, M.; Pinget, M.; Jeandidier, N.; Vauthier, C.; Marchioni, E.; Frere, Y.;Sigrist, S. Design, characterisation, and bioefficiency of insulin-chitosan nanoparticles after stabilisation by freezedrying or cross-linking. International Journal of Pharmaceutics 2015, 491, 402-408, https://doi.org/10.1016/j.ijpharm.2015.05.065.

41. Ramalingam, P.; Ko, Y.T.; Enhanced oral delivery of curcumin from N-trimethyl chitosan surface-modified solid lipid nanoparticles: pharmacokinetic and brain distribution evaluations. Pharmaceutical research 2015, 32, 389-402, https://doi.org/10.1007/s11095-014-1469-1.

42. Kulkarni, A.D.; Joshi, A.A.; Patil, C.L.; Amale, P.D.; Patel, H.M.; Surana, S.J.; Belgamwar, V.S.; Chaudhari, K.S.; Pardeshi, C.V.; Xyloglucan: A functional biomacromolecule for drug delivery applications. International Journal of Biological Macromolecule 2017, 104, 799-812, https://doi.org/10.1016/j.ijbiomac.2017.06.088.

43. Han, M.; Liu, Y.; Zhang, F.; Sun, D.; Jiang, J. Effect of galactose side-chain on the self-assembly of xyloglucan macromolecule. Carbohydr. Polym. 2020, 246, 116577 , https://doi.org/10.1016/j.carbpol.2020.116577.

44. Da Silva, A.C.C.; De Almeida, R.R.; Da Cruz Sousa, A.C.; Martinez, F.N.A.; Denardin, J.C.; De Morais, S.M.; Ricardo, N.M.P.S. Xyloglucan-based hybrid nanocomposite with potential for biomedical applications. International Journal of Biological Macromolecule 2021, 168, 722-732, https://doi.org/10.1016/j.ijbiomac.2020.11.128.

45. Chandrakantsing V, Pardeshi.; Abhijeet D, Kulkarni.; Veena S, Belgamwar.; Sanjay J, Surana. Chapter 7 Xyloglucan for drug delivery applications. Fundamental Biomaterials: Polymers, Sabu Thomas, Preetha Balakrishnan, M.S. Sreekala.; In Woodhead Publishing Series inBiomaterials: India 2018,143-169, https://doi.org/10.1016/B978-0-08-102194-1.00007-4.

46. Hummers, W.S., Offeman, R.E. Preparation of Graphitic Oxide. Journal of the American Chemical Society 1958, 80, 1339-1339, https://doi.org/10.1021/ja01539a017.

47. Marcano, D.C.; Kosynkin, D.V.; Berlin, J.M.; Sinitskii, A.; Sun, Z.; Slesarev, A.; Alemany, L.B.; Lu,W.;Tour, J.M. Improved synthesis of graphene oxide. ACS Nano 2010, 4, 4806-4814, https://doi.org/10.1021/nn1006368. 
48. Muthukumarasamyvel, T.; Baskar, R.; Chandirasekar, S.; Umamaheswari, K.; Rajendiran, N.Hierarchical self- assembly of bile-acid-derived dicationic amphiphiles and their toxicity assessment on microbial and mammalian systems. ACS Applied Materials and Interfaces 2016, 8, 25111- 25126, https://doi.org/10.1021/acsami.6b08018.

49. Paarakh, M.P.; Jose, P.A.; Setty, C.; Christoper, G. Release kinetics-concepts and applications. International Journal of Pharmacy Research and Technology 2018, 8, 12-20, https://doi:10.31838/ijprt/08.01.02.

50. Kumar, N.; Srivastava, V.C. Simple Synthesis of Large Graphene Oxide Sheets via Electrochemical Method Coupled with Oxidation Process. ACS Omega 2018, 3, 10233-10242, https://doi.org/10.1021/acsomega.8b01283.

51. Su, Z.; Sun, D.; Zhang, L.; He, M.; Jiang, Y.; Millar, B.; Douglas, P.; Mariotti, D.; Maguire, P.; Sun, D. Chitosan/Silver Nanoparticle/Graphene Oxide Nanocomposites with Multi-Drug Release, Antimicrobial, and Photothermal Conversion Functions. Materials 2021, 14, 2351, https://oi.org/10.3390/ma14092351.

52. Aliyev, E.; Filiz, V.; Khan, M.M.; Lee, Y.J.; Abetz, C.; Abetz, V. Structural characterization of Graphene Oxide: surface functional groups and fractionated oxidative debris. Nanomaterials 2019, 9, 1180, https://doi.org/10.3390/nano9081180.

53. López-Díaz, D.; Delgado-Notario, J.A.; Clericò, V.; Diez, E.; Merchán, M.D.; Velázquez, M.M.;Towards Understanding the Raman Spectrum of Graphene Oxide: The Effect of the Chemical Composition. Coatings 2020, 10,524, https://doi.org/10.3390/coatings 10060524.

54. Shu, M.; Gao, F.; Zeng, M.; Yu, C.; Wang, X.; Huang, R.; Yang, J.; Su, Y.; Hu, N.; Zhou, Z.; Liu,K.;Yang, Zhi.;T an, H.; Xu, L. et al. Microwave-Assisted Chitosan-Functionalized Graphene Oxide as Controlled Intracellular Drug Delivery Nanosystem for Synergistic Antitumour Activity. Nanoscale Res Lett 2021, 16, 75, https://doi.org/10.1186/s11671-021-03525-y.

55. Mhlanga, N.; Ray, S.S. Kinetic models for the release of the anticancer drug doxorubicin from biodegradable polylactide/metal oxide-based hybrids. International Journal of Biological Macromolecule 2015, 72, 13011307, https://doi.org/10.1016/j.ijbiomac.2014.10.038.

56. Prasanth, A.G.; Kumar, A.S.; Shruthi, B.S.; Subramanian, S. Kinetic study and in vitro drug release studies of nitrendipine loaded arylamide grafted chitosan blend microspheres. Materials Research Express 2020, 5 , 125427, https://doi.org/10.1088/2053-1591/ab5811. 